\title{
Exposure to Adipose-Enriched Bone Marrow Increased Breast Cancer Cell Expression of Genes that Drive Tumor Metastasis
}

\author{
Laura E. Wright, ${ }^{1}$ Sukanya Suresh, ${ }^{1}$ Jenna N. Regan, ${ }^{2}$ Gabriel M. Pagnotti, ${ }^{1}$ \\ Sutha John, ${ }^{1}$ Khalid S. Mohammad, ${ }^{1}$ Theresa A. Guise ${ }^{1}$ \\ ${ }^{1}$ Indiana University School of Medicine, Department of Medicine, Division of \\ Endocrinology, Indianapolis IN, USA. \\ ${ }^{2}$ University of Illinois College of Medicine at Peoria, Department of Health \\ Sciences Education, Peoria, IL.
}

Background and Hypothesis: Previously thought to be inert, bone marrow adipose tissue (BMAT) has emerged as a significant fat depot that increases with age, radiation, estrogen deficiency, and high fat diet. Because bone is a preferred site for breast cancer metastasis, the lack of understanding regarding the effects of BMAT accrual on tumor cell migration and proliferation represents a significant knowledge gap in the cancer field. We hypothesized that exposure to adipokines secreted from BMAT-enriched bone marrow would induce changes in the tumor cell transcriptome.

Experimental Design or Project Methods: Twenty-week female C57BI/6 mice were randomized and treated with control diet (5\% kcal\% fat) or high fat diet (HFD; $60 \%$ kcal\% fat) for nine weeks. Relative to control mice, HFD mice had increased body weight, peripheral fat mass, BMAT volume, and circulating adipokines. Bone marrow was flushed and cultured for 24 hours, and control or BMAT-enriched conditioned media was collected. The adipokines leptin and MCP-1 were assayed by ELISA and found to be increased in media obtained from BMAT-enriched cultures. Human MDAMB-231 breast cancer cells were then cultured in bone marrow conditioned media for 24 hours and tumor cells were processed for RNA sequencing.

Results: Exposure to soluble factors derived from BMAT-enriched cultures resulted in significant increase in the expression of genes associated with cancer cell motility and neovascularization, including SEMA4C, PLXNA1, and VEGFA.

Conclusion and Potential Impact: These data indicate that BMAT contributes to the tumor microenvironment and may drive the progression of breast cancer bone metastases. 